TRABAJO EN PROGRESO

\title{
Evaluación de la experiencia de los usuarios de un quiosco informativo en un parque público
}

\author{
Arturo García, Guillermo Arcos, Jonathan S. \\ Ramírez, Isaac Jaramillo, Pedro C. Santana- \\ Mancilla, Roberto D. Ochoa, Víctor H. Medina
}

Publicado: 21 Septiembre 2016

\begin{abstract}
Resumen
Este artículo presenta una propuesta de prototipo de un quiosco informativo con mapa interactivo que muestra al visitante información acerca de los lugares de interés en un parque público de la ciudad de Villa de Álvarez. El mapa interactivo es una interfaz que incorpora información comercial de los negocios locales, así como la organización espacial de la zona. El visitante puede acceder a instrucciones que le permiten usar el mapa, y observar información respecto de los negocios que le interesen, de manera que pueda planear actividades con anticipación, y mejorar su experiencia de visita. Se realizó una evaluación de usabilidad para determinar el nivel en que los usuarios pueden utilizar el mapa interactivo propuesto. Además de evaluar la aceptación de dicho prototipo por parte de los usuarios.
\end{abstract}

Palabras clave: Experiencia del usuario; Pruebas de usabilidad.

\section{Introducción}

La información oportuna, actual y disponible en cualquier momento para toda persona interesada en ella, son características que, en estos tiempos todo usuario necesita en cualquier ámbito. Tomando esta premisa, se desarrolló la propuesta de otorgar a los visitantes de un parque público, información útil y oportuna sobre sitios que fueran de su interés dentro del mismo por medio de un quiosco informativo.

El presente trabajo recopila la evaluación de la propuesta de un quiosco informativo que proporcione a los visitantes del parque, la información de los principales establecimientos y puntos de interés que se encuentran en él.

\subsection{Mapa interactivo para parque público}

El quiosco informativo consta de una base y de una pantalla, la cual se divide en dos secciones. La primera muestra el mapa de la ubicación (ver Figura 1), y la segunda muestra las instrucciones de uso, así como la información que el usuario solicita. Se

García, A., Arcos, G., Ramírez, JS., Jaramillo, I., Santana-Mancilla, PC., Ochoa, RD., Medina, VH.

Facultad de Telemática Universidad de Colima, Av. Universidad 333, Colima, México

Email: \{narturo, guillermo_arcos, jrcovarrubias, isaak, psantana, rochoa5, vmedina\}@ucol.mx construyó pensando en su posible ubicación estratégica en varios puntos de un parque público, de manera que varios visitantes puedan acceder al sistema de manera simultánea. La interfaz divide la pantalla en dos partes, de manera que el usuario pueda localizar los negocios que se encuentren dentro del parque, y obtener información acerca de los mismos.

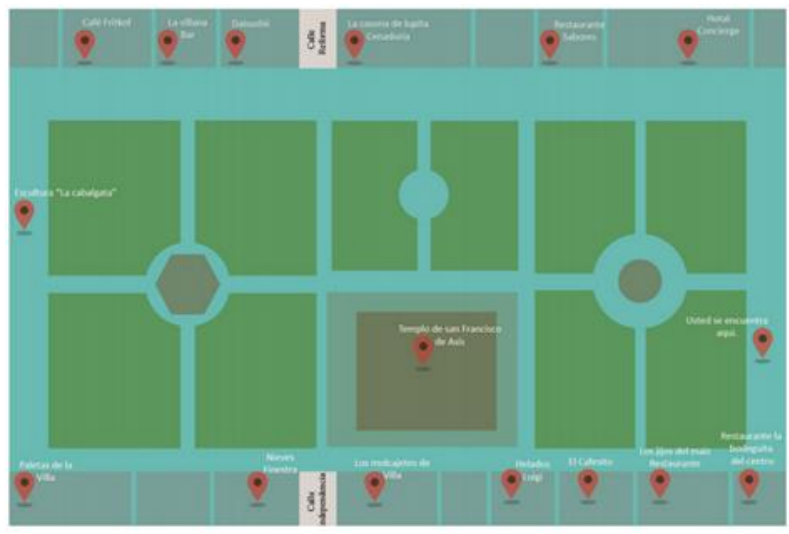

Figura 1. Mapa interactivo

Para medir la experiencia de los usuarios se utilizó el Modelo de Aceptación Tecnológico (TAM, por sus siglas en inglés Technology Acceptance Model) [1] y la Escala de Usabilidad de los Sistemas (SUS, por sus siglas en inglés System Usability Scale) [2].

\section{Metodología}

\subsection{Lugar}

Para la evaluación del prototipo diseñado, se abordaron a seis jóvenes visitantes de un parque público en la ciudad de Villa de Álvarez en el estado de Colima, entre estos jóvenes se encontraban cuatro mujeres y dos hombres con un promedio de edad entre los 16 y los 26 años de edad.

\subsection{Proceso}

Antes de que los usuarios realizaran las pruebas de usabilidad se aplicó una pequeña encuesta donde se preguntaba la frecuencia con la que visitaban el parque, así como los motivos y actividades que realizaban en dicho lugar, también se les preguntó si en algún 
momento habían utilizado un sistema con características similares al presentado en el prototipo.

Se continuó con la prueba del prototipo donde a los usuarios se les dio una lista de tareas que debían realizar con el quiosco informativo, posterior al uso del dispositivo se aplicaron los cuestionarios TAM y SUS.

\section{Evaluación}

Para poder medir la aceptación, la percepción de utilidad y facilidad de uso del prototipo, se realizaron las pruebas de usabilidad correspondientes en un ambiente real.

Como se mencionó previamente, estas mediciones se realizaron a través de 3 principales instrumentos, la Escala de Usabilidad de Sistemas (SUS), Modelo de Aceptación de Tecnología (TAM), observaciones durante las pruebas con los usuarios y entrevistas finales posteriores a las pruebas. Los resultados de estos instrumentos se presentan a continuación.

\subsection{Aceptación de la tecnología}

Para medir la aceptación de la tecnología del sistema propuesto se utilizó el Modelo de Aceptación de la Tecnología (TAM). El propósito del TAM es explicar las causas de la aceptación de la tecnología por parte del usuario. Además, propone que la percepción de utilidad y facilidad de uso del usuario en un sistema de información son concluyentes para determinar su intención de utilizar el sistema.

Se usaron muestras de 6 personas, las cuales participaron en utilizar el prototipo. Fueron 4 mujeres y 2 hombres. Todas las personas participantes se encontraron en parque público, en este caso el jardín principal de la ciudad de Villa de Álvarez llamado Independencia.

Tomando en cuenta las respuestas "totalmente de acuerdo" del instrumento TAM, los resultados que arrojaron las pruebas fueron los siguientes:

Para el punto de la Percepción de Facilidad de Uso el 83.3\% de los usuarios evaluados consideran que es fácil de usar, fácil de aprender, claro y entendible. El mismo porcentaje coincide en que es fácil de navegar.

En el punto de Percepción de Utilidad, $83.3 \%$ de los usuarios evaluados creen que el software es eficaz, que mejorarán su desempeño haciendo uso de él, de igual manera que mejorarán su productividad y que es muy útil.

En el punto de Actitud hacia Uso del Software, se observó que el $83.3 \%$ no le disgusta la idea de usar el software mientras que al $100 \%$ tiene una actitud favorable hacia el uso del mismo, creen que es una buena idea y no piensan que sea una idea tonta.

Para el punto de Intención de Uso el 100\% tiene intención de volver a usar el software y que lo van a volver a usar, el mismo porcentaje opina que se debe de usar para sus visitas al parque.

\subsection{Usabilidad}

La escala SUS ha demostrado dar un valor confiable y simple para determinar qué tan usable es un sistema de acuerdo a la perspectiva del usuario que la utiliza.

El usuario contesta el cuestionario SUS única y exclusivamente después de haber interactuado con el sistema, cabe mencionar que para que este instrumento arroje los resultados correctos, los usuarios no debieron de haber interactuado con la interfaz a evaluar en ocasiones anteriores a la que se evalúa.

De cada cuestionario que se aplicó se tiene una escala de resultados que va de 0 (nula usabilidad) a 100 (muy alta usabilidad). El promedio de los cuestionarios aplicados para la evaluación del quiosco informativo del Jardín de Villa de Álvarez, fue de 87.08 puntos por lo que de acuerdo a la teoría [3] puede ser considerado como muy alta usabilidad.

La distribución de frecuencias (ver Figura 2) nos muestra que el $16.67 \%$ de los usuarios calificaron la interfaz con un valor menor a 70 puntos, el $50 \%$ de los usuarios calificaron la interfaz con un valor entre 81 y 90 y el $33.34 \%$ con un valor mayor a 91 puntos.

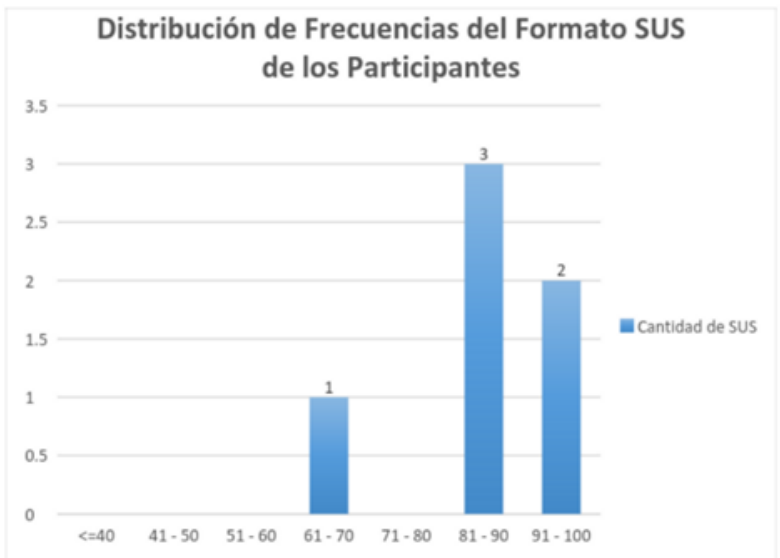

Figura 2. Distribución de frecuencias SUS de los usuarios

Esto es un buen resultado porque hablando de evaluaciones SUS, un valor mínimo de 68 puntos predice que el prototipo tiene una usabilidad adecuada y analizando los resultados de estas pruebas, podemos inferir que el sistema goza de una muy alta usabilidad.

\section{Conclusiones}

Este trabajo presenta el prototipo de un quiosco informativo que muestra a los visitantes de un parque público información acerca de los lugares de interés dentro del mismo.

Se puede concluir con la Escala de Usabilidad de Sistemas (SUS) se tiene una interfaz con muy alta usabilidad, por lo cual existe una probabilidad alta de que los mismos usuarios promuevan el uso de este quiosco. Asimismo la prueba TAM permitió observar un muy buen grado de aceptación de la tecnología propuesta.

Con base en las pruebas descritas en este reporte y al interés observado en los usuarios es posible dictaminar que es un sistema usable y completa en su totalidad los objetivos esperados de este.

\section{Referencias}

[1] Morris, M., \& Dillon, A. (1997, August). How user perceptions influence software use. IEEE Software, 14(4), 58-65. doi:10.1109/52.595956

[2] Veneziano, V., \& Mahmud, I. (2014, December 20). Usability analysis of ERP software: Education and experience of users' as moderators. InSoftware, Knowledge, Information Management and Applications (SKIMA), 2014 8th International Conference on. Retrieved July 08, 2016.

[3] Lewis, J. R., \& Sauro, J. (2011). The Factor Structure of the System Usability Scale . Boca Raton, Florida, Estados Unidos de América. 